2017

\title{
A Madison-Numeracy Citation Index (2008-2015): Implementing a Vision for a Quantitatively Literate World
}

Nathan D. Grawe

Carleton College, ngrawe@carleton.edu

H. L. Vacher

University of South Florida, vacher@usf.edu

Follow this and additional works at: https://digitalcommons.usf.edu/numeracy

Part of the Curriculum and Instruction Commons, and the Other Mathematics Commons

\section{Recommended Citation}

Grawe, Nathan D., and H. L. Vacher. "A Madison-Numeracy Citation Index (2008-2015): Implementing a Vision for a Quantitatively Literate World." Numeracy 10, Iss. 1 (2017): Article 1. DOI: http://dx.doi.org/ 10.5038/1936-4660.10.1.1 


\title{
A Madison-Numeracy Citation Index (2008-2015): Implementing a Vision for a Quantitatively Literate World
}

\begin{abstract}
This editorial recognizes the contributions made by Bernard Madison to the field of quantitative literacy with a bibliographic index of his papers, edited volumes, and works contained therein that were cited in the first eight volumes (2008-2015) of Numeracy. In total, 61 citing papers ("sources") cite 42 Madison works ("citations") a total of 218 times. The source and citation indexes provided in the appendix at the end of this editorial make it easy to see the direct contribution of Madison's work to the arguments and debates contained in the founding years of the journal. For those who are new to the field of quantitative reasoning, the citation index also provides an essential reading list. Most of the citations and sources are open-access and links within the indexes aid easy access to Madison's important contribution to Numeracy and the quantitative literacy movement.
\end{abstract}

\section{Keywords}

mathematicians, bibliography, quantitative literacy, bibliometrics, University of Arkansas

\section{Creative Commons License}

(c) (1) (9)

This work is licensed under a Creative Commons Attribution-Noncommercial 4.0 License

\section{Cover Page Footnote}

Nathan D. Grawe is Professor and Chair of Economics at Carleton College. His involvement in quantitative reasoning has focused on assessment and faculty development. He is a co-editor of this journal.

Len Vacher is Professor of Geology in the School of Geosciences at the University of South Florida. He is a founding co-editor of this journal. 
Readers may recall last volume's lead-off editorial in memory of Lynn Steen (Vacher 2015). That piece quantified the impact of Steen's visionary work on our field through works cited in papers published in Numeracy's first eight volumes (2008-2015). Even a cursory read of the Steen-Numeracy Citation Index reveals the crucial role played by Bernard (Bernie) Madison as co-author and co-editor. For instance, of the 67 Steen works cited in Numeracy papers, 40 percent were joint ventures with Madison. Even more to the point, 11 of the 14 most-cited Steen works in Numeracy's first eight years might more accurately be classified "Madison-Steen works." Given the potency of this research partnership and Madison's individual contribution through the influence of works on which Steen is not a co-author, we thought it appropriate to begin this $10^{\text {th }}$ volume of Numeracy by honoring the contributions of Bernie Madison as seen through the lens of a companion Madison-Numeracy Citation index.

The citation index, which is in the Appendix below, contains two parts: (1) a citation list ("The Cited References") identifying all of the Madison works cited in Numeracy papers in the years 2008 through 2015, and (2) a source list section ("The Citing Papers") naming all of the Numeracy works that cite the Madison works in the citation list. The citing papers in Part 2 provide evidence of the wide influence that Madison's published works have had on the growth of our discipline; for example, of the 151 articles, perspective pieces, reviews, columns, and editorials published in the first eight volumes of this journal, 61 (more than 40 percent) explicitly connect their work to Madison's work through citation. (By personal experience, we attest that this is surely an underestimate of that influence as other Numeracy papers bear the marks of Bernie's work despite lacking a citation.) Taken together with the 10 Numeracy works written or co-authored by Madison, these data speak clearly to his indelible mark on the early years of the journal.

Citation patterns of the cited papers provide a window into just what it is in Madison's work that so many of us have found important. In total, the 42 Madison works listed in Part 1 are cited 218 times by the citing articles contained in Part 2. Of these citations, 171 (78\%) point to just 14 of the Madison works. These are listed in Table 1 ordered by the number of times each was cited. Vacher (2015) argues that Steen's work made the case for quantitative literacy (QL). Evident in the non-shaded items in Table 1, Madison was a trusted collaborator in this intellectual effort. However, the shaded entries in Table 1 point to an important second thrust in Bernie's publications: Madison was a trailblazer in developing and teaching QL curricula. In these pieces authored alone or with co-authors other than Steen, Madison shared his experiences in the classroom attempting to implement the vision he developed along with Steen. This work represents a critical inflection point in any movement - the shift from hypothesizing to experimenting, from talking to doing. What is more, Madison 
shared casebook materials and reflections in this journal and elsewhere so that others could cut their own paths with greater efficiency and effect.

Table 1.

The 14 Most-Cited Works from the Citation List of Part 1 of the Appendix

\begin{tabular}{|c|c|c|c|c|}
\hline $\begin{array}{l}\text { Rank } \\
\text { Order }\end{array}$ & Citations & Title & Reference & Type \\
\hline 1 & 29 & $\begin{array}{l}\text { Quantitative literacy: Why numeracy matters for } \\
\text { schools and colleges }\end{array}$ & $\begin{array}{l}\text { Madison and } \\
\text { Steen } 2003\end{array}$ & Edited volume \\
\hline 2 & 27 & $\begin{array}{l}\text { Calculation vs. context: Quantitative literacy and its } \\
\text { implications for teacher education }\end{array}$ & $\begin{array}{l}\text { Madison and } \\
\text { Steen } 2008 b\end{array}$ & Edited volume \\
\hline 3 & 22 & $\begin{array}{l}\text { Case studies for quantitative reasoning: A casebook of } \\
\text { media articles }\end{array}$ & $\begin{array}{l}\text { Madison and } \\
\text { Dingman } 2008 \\
\text { and Madison et } \\
\text { al. } 2009\end{array}$ & Book \\
\hline 4 & 19 & $\begin{array}{l}\text { Evolution of numeracy and the National_Numeracy } \\
\text { Network }\end{array}$ & $\begin{array}{l}\text { Madison and } \\
\text { Steen 2008a }\end{array}$ & $\begin{array}{l}\text { Article in } \\
\text { Numeracy }\end{array}$ \\
\hline 5 & 11 & $\begin{array}{l}\text { Quantitative Reasoning in the Contemporary World, } 1 \text { : } \\
\text { The course and its challenges }\end{array}$ & $\begin{array}{l}\text { Dingman and } \\
\text { Madison } 2010\end{array}$ & $\begin{array}{l}\text { Article in } \\
\text { Numeracy }\end{array}$ \\
\hline 6 & 10 & $\begin{array}{l}\text { Arguing with numbers: Teaching quantitative reasoning } \\
\text { through argument and writing }\end{array}$ & Lutsky 2008 & $\begin{array}{l}\text { Article in Madison } \\
\text { and Steen 2008b }\end{array}$ \\
\hline 7 & 9 & $\begin{array}{l}\text { Quantitative Reasoning in the Contemporary World, } 2 \\
\text { Focus questions for the numeracy community }\end{array}$ & $\begin{array}{l}\text { Madison and } \\
\text { Dingman } 2010\end{array}$ & $\begin{array}{l}\text { Article in } \\
\text { Numeracy }\end{array}$ \\
\hline 8 & 8 & The third $R$ in literacy & $\begin{array}{l}\text { Richardson and } \\
\text { McCallum } 2003\end{array}$ & $\begin{array}{l}\text { Article in Madison } \\
\text { and Steen } 2003\end{array}$ \\
\hline 8 & 7 & $\begin{array}{l}\text { Reflections on quantitative reasoning: An assessment } \\
\text { perspective }\end{array}$ & Shavelson 2008 & $\begin{array}{l}\text { Article in Madison } \\
\text { and Steen 2008b }\end{array}$ \\
\hline 9 & 7 & $\begin{array}{l}\text { Preparing students for the business of the real (and } \\
\text { highly quantitative) world }\end{array}$ & Taylor 2008 & $\begin{array}{l}\text { Article in Madison } \\
\text { and Steen } 2008 b\end{array}$ \\
\hline 11 & 6 & $\begin{array}{l}\text { Quantitative Reasoning in the Contemporary World, } 3 \text { : } \\
\text { Assessing student learning }\end{array}$ & $\begin{array}{l}\text { Boersma et al. } \\
2011\end{array}$ & $\begin{array}{l}\text { Article in } \\
\text { Numeracy }\end{array}$ \\
\hline 11 & 6 & $\begin{array}{l}\text { Quantitative literacy and school mathematics: } \\
\text { Percentages and fractions }\end{array}$ & Schield 2008 & $\begin{array}{l}\text { Article in Madison } \\
\text { and Steen } 2008 b\end{array}$ \\
\hline 13 & 5 & $\begin{array}{l}\text { The role of mathematics courses in the development of } \\
\text { quantitative literacy }\end{array}$ & $\begin{array}{l}\text { Hughes-Hallett } \\
2003\end{array}$ & $\begin{array}{l}\text { Article in Madison } \\
\text { and Steen } 2003\end{array}$ \\
\hline 13 & 5 & Quantitative literacy: Everybody's orphan & Madison 2001 & $\begin{array}{l}\text { Article in } M A A \\
\text { Focus }\end{array}$ \\
\hline
\end{tabular}

In many aspects of his work, Madison has been a vital and dependable bridge. First, when the National Numeracy Network emerged from the justcompleted National Council on Education and the Disciplines, it was Bernie who served as its first president and led the organization in its transformation into a national membership association. Second, even as he took the lead in developing alternatives to the traditional, calculus-oriented mathematics curriculum he simultaneously worked to improve that traditional curriculum through service on the AP Calculus Committee (and as Chief Reader from 1995 to 1999). And finally (from Table 1), Bernie "went first" putting into practice the visionary ideals of quantitative literacy articulated by Steen and other educational theorists (including himself). The important characteristic of bridges is that they stand with firm footing on each of the two sides of the gulf. Finding ways to bring two 
different lands together can be complex work, often accomplished at a personal cost. Those who have worked with him can attest that Bernie took on these challenges with good will, good humor, and good sense.

For those who are new to the quantitative literacy movement and are looking to get up to speed, you could do a lot worse than visit the 23 most-cited works in the Steen-Numeracy and Madison-Numeracy citations lists (Table 1 here and in Vacher 2015). But if you want to fully understand those pieces and their intellectual progeny, be prepared to read a while. Between them, these works have been cited more than 269 times in Numeracy alone. Happy reading!

\section{Reference}

Vacher, H. L. 2015. Remembering Lynn Steen: A Steen-Numeracy citation index (2008-2015). Numeracy 9(1): Article 1. http://dx.doi.org/10.5038/1936$\underline{4660.9 .1 .1}$

\section{Appendix}

\section{Madison-Numeracy Citation Index (2008-2015) Compiled by Len Vacher (Jan 2016) \\ Part 1: Citation List (The Cited References)}

Bass, Hyman. 2003. "What Have We Learned ... and What Have We Yet to Learn?" In Madison and Steen 2003: 247-249.

Madison and Steen $2009 \quad$ (http://dx.doi.org/10.5038/1936-4660.2.1.2)

Best, Joel. 2008. "Beyond Calculation: Quantitative Literacy and Critical Thinking About Public Issues." In Madison and Steen 2008: 125-135.

Vacher and Lardner $2010 \quad$ (http://dx.doi.org/10.5038/1936-4660.3.2.6)

Madison $2012 \quad$ (http://dx.doi.org/10.5038/1936-4660.5.1.6)

Boersma, Stuart, Caren Diefenderfer, Shannon W. Dingman, and Bernard L. Madison. 2011. "Quantitative Reasoning in the Contemporary World, 3: Assessing Student Learning." Numeracy 4 (2), Article 8.

Boersma and Klyve 2013a $\quad$ http://dx.doi.org/10.5038/1936-4660.6.1.5) Boersma and Klyve 2013b (http://dx.doi.org/10.5038/1936-4660.6.1.6) Grawe $2013 \quad$ (http://dx.doi.org/10.5038/1936-4660.6.2.11) Vacher $2014 \quad$ (http://dx.doi.org/10.5038/1936-4660.7.2.1) Madison 2014 (http://dx.doi.org/10.5038/1936-4660.7.2.3) Russo 2015 (http://dx.doi.org/10.5038/1936-4660.8.1.8)

Brakke, David F. 2003. “Addressing Societal and Workforce Needs.” In Madison and Steen 2003: 167-169.

Grawe and Rutz 2009 (http://dx.doi.org/10.5038/1936-4660.2.2.2) 
Burkhardt, Hugh. 2008. "Quantitative Literacy for All: How Can We Make It Happen?” In Madison and Steen 2008: 137-162.

$\begin{array}{ll}\text { Madison } 2012 & \text { (http://dx.doi.org/10.5038/1936-4660.5.1.6) } \\ \text { Tunstall and Bossé 2015 } & \text { (http://dx.doi.org/10.5038/1936-4660.8.2.10) }\end{array}$

Carnevale, Anothy P. and Donna M. Desrochers. 2003. "The Democratization of Mathematics." In Madison and Steen 2003: 21-30.

Steen and Madison $2011 \quad$ (http://dx.doi.org/10.5038/1936-4660.4.1.1)

Todd and Wagaman $2015 \quad$ (http://dx.doi.org/10.5038/1936-4660.8.2.9)

Cohen, Patricia Cline. 2003. "Democracy and the Numerate Citizen: Quantitative Literacy in Historical Perspective." In Madison and Steen 2003: 7-20.

Todd and Wagaman $2015 \quad$ (http://dx.doi.org/10.5038/1936-4660.8.2.9)

Colwell, Rita. 2003. “Quantitative Literacy Goals: Are We Making Progress?” In Madison and Steen 2003: 243-246.

Tunstall and Bossé 2015 (http://dx.doi.org/10.5038/1936-4660.8.2.10)

De Lange, Jan. 2003. “Mathematics for Literacy.” In Madison and Steen 2003: 75-89.

Grawe et al. 2010

Kosko and Wilkins 2011

Smit and Mji 2012

Todd and Wagaman 2015 (http://dx.doi.org/10.5038/1936-4660.3.1.3)

(http://dx.doi.org/10.5038/1936-4660.4.2.3)

(http://dx.doi.org/10.5038/1936-4660.5.2.4)

(http://dx.doi.org/10.5038/1936-4660.8.2.9)

Dingman, Shannon W., and Bernard L. Madison. 2010. "Quantitative Reasoning in the Contemporary World, 1: The Course and Its Challenges." Numeracy 3 (2), Article 4.

Madison and Dingman 2010

Steen and Madison 2011

Boersma et al. 2011

Frith 2012

Madison 2012

Boersma and Klyve 2013a

Rocconi et al. 2013

Dumford and Rocconi 2015

Russo 2015

Tunstall 2015

Ricchezza and Vacher 2015. (http://dx.doi.org/10.5038/1936-4660.3.2.5) (http://dx.doi.org/10.5038/1936-4660.4.1.1) (http://dx.doi.org/10.5038/1936-4660.4.2.8) (http://dx.doi.org/10.5038/1936-4660.5.1.3) (http://dx.doi.org/10.5038/1936-4660.5.1.6) http://dx.doi.org/10.5038/1936-4660.6.1.5) (http://dx.doi.org/10.5038/1936-4660.6.2.10) (http://dx.doi.org/10.5038/1936-4660.8.1.5) (http://dx.doi.org/10.5038/1936-4660.8.1.8) (http://dx.doi.org/10.5038/1936-4660.8.2.12) (http://dx.doi.org/10.5038/1936-4660.8.2.14)

Hughes-Hallett, Deborah. 2003. "The Role of Mathematics Courses in the Development of Quantitative Literacy." In Madison and Steen 2003: 91-98.

Steele and Kiliç-Bahi 2008

Grawe and Rutz 2009 (http://dx.doi.org/10.5038/1936-4660.1.2.3)

(http://dx.doi.org/10.5038/1936-4660.2.2.2) 
Sikorskii et al. 2011

Sundre et al. 2012

Tunstall and Bossé 2015 (http://dx.doi.org/10.5038/1936-4660.4.2.5)

(http://dx.doi.org/10.5038/1936-4660.5.1.4)

(http://dx.doi.org/10.5038/1936-4660.8.2.10)

Lutsky, Neil. 2008. "Arguing with Numbers: Teaching Quantitative Reasoning Through Argument and Writing." In Madison and Steen 2008: 59-74.

Vacher and Chavez 2008

(http://dx.doi.org/10.5038/1936-4660.1.2.2)

Madison and Steen 2009

(http://dx.doi.org/10.5038/1936-4660.2.1.2)

Lutsky 2009

Grawe et al. 2010

(http://dx.doi.org/10.5038/1936-4660.2.1.6)

Madison 2012

(http://dx.doi.org/10.5038/1936-4660.3.1.3)

Hillyard 2012

(http://dx.doi.org/10.5038/1936-4660.5.1.6)

Polito 2014

(http://dx.doi.org/10.5038/1936-4660.5.2.2)

Wright and Howard 2015

(http://dx.doi.org/10.5038/1936-4660.7.1.6)

(http://dx.doi.org/10.5038/1936-4660.8.1.6)

Follette et al. 2015

Tunstall and Bossé 2015

(http://dx.doi.org/10.5038/1936-4660.8.2.2)

(http://dx.doi.org/10.5038/1936-4660.8.2.10)

Madison, B. 2001. “Quantitative Literacy: Everybody's Orphan.” MAA Focus 21 (6): 10-11.

Mast 2009 (http://dx.doi.org/10.5038/1936-4660.2.2.6)

Maclellan 2012

Madison 2012

Madison 2014

(http://dx.doi.org/10.5038/1936-4660.5.2.3)

(http://dx.doi.org/10.5038/1936-4660.5.1.6)

(http://dx.doi.org/10.5038/1936-4660.7.2.3)

Ricchezza and Vacher 2015. ～(http://dx.doi.org/10.5038/1936-4660.8.2.14)

Madison, Bernard. 2002. "Educating for Numeracy: A Challenging

Responsibility." Notices of the American Mathematical Society (February): 181

Madison and Steen 2009

(http://dx.doi.org/10.5038/1936-4660.2.1.2)

Madison, Bernard L. 2003. "Articulation and Quantitative Literacy: A View from

Inside Mathematics." In Madison and Steen 2003: 153-164.

Rheinlander et al. $2008 \quad$ (http://dx.doi.org/10.5038/1936-4660.1.1.4)

Mayes et al. $2013 \quad$ (http://dx.doi.org/10.5038/1936-4660.6.1.4)

Madison $2014 \quad$ (http://dx.doi.org/10.5038/1936-4660.7.2.3)

Tunstall and Bossé $2015 \quad$ (http://dx.doi.org/10.5038/1936-4660.8.2.10)

Madison, Bernard L. 2004. “Two Mathematics: Ever the Twain Shall Meet?” Peer Review 6(4): 9-12.

Madison $2009 \quad$ (http://dx.doi.org/10.5038/1936-4660.2.1.1)

Madison, Bernard. 2006. "Preparing for Calculus and Preparing for Life." In $A$

Fresh Start for Collegiate Mathematics: Rethinking the Courses Below Calculus, ed. Nancy Baxter Hastings et al., 78-21. Washington D.C.: Mathematical

Association of America.

Madison and Steen $2009 \quad$ (http://dx.doi.org/10.5038/1936-4660.2.1.2) 
Madison, Bernard. 2006. "Pedagogical Challenges of Quantitative Literacy." Proceedings of the Joint Statistical Meetings, 2323-2328. Alexandria, VA: American Statistical Association.

$\begin{array}{ll}\text { Mayes et al. 2013 } & \text { (http://dx.doi.org/10.5038/1936-4660.6.1.4) } \\ \text { Dingman and Madison 2010 } & \text { (http://dx.doi.org/10.5038/1936-4660.3.2.4) } \\ \text { Russo 2015 } & \text { (http://dx.doi.org/10.5038/1936-4660.8.1.8) }\end{array}$

Madison, Bernard. 2007. "Mathematical Proficiency for Citizenship." In Assessing Mathematical Proficiency, ed. Alan H. Schoenfeld, 113-125. New York: Cambridge University Press. Madison and Steen $2009 \quad$ (http://dx.doi.org/10.5038/1936-4660.2.1.2)

Madison, Bernard L. 2009. "All the More Reason for QR Across the Curriculum." Numeracy 2 (1), Article 1.

Steele and Kiliç-Bahi $2010 \quad$ (http://dx.doi.org/10.5038/1936-4660.3.2.3)

Hillyard 2012

Rocconi et al. 2013

(http://dx.doi.org/10.5038/1936-4660.5.2.2)

Dumford and Rocconi $2015 \quad$ (http://dx.doi.org/10.5038/1936-4660.8.1.5)

Madison, Bernard L. 2012. "If Only Math Majors Could Write ...." Numeracy 5 (1), Article 6.

Grawe 2013 (http://dx.doi.org/10.5038/1936-4660.6.2.11)

Polito $2014 \quad$ (http://dx.doi.org/10.5038/1936-4660.7.1.6)

Wang and Wilder $2015 \quad$ (http://dx.doi.org/10.5038/1936-4660.8.2.6)

Madison, Bernard L. 2014. "How Does One Design or Evaluate a Course in Quantitative Reasoning?" Numeracy 7 (2), Article 3.

Howington et al. $2015 \quad$ (http://dx.doi.org/10.5038/1936-4660.8.1.10)

Madison, Bernard L. 2015. "Quantitative Literacy and the Common Core

Standards in Mathematics." Numeracy 8 (1), Article 11.

Whitaker et al. $2015 \quad$ (http://dx.doi.org/10.5038/1936-4660.8.2.3)

Madison Bernard and Shannon Dingman. 2008. Case Studies for Quantitative

Reasoning: A Casebook of Media Articles. Pearson Custom Publishing Madison and Steen $2009 \quad$ (http://dx.doi.org/10.5038/1936-4660.2.1.2)

Madison, B., and S. Dingman. 2010. "Quantitative Reasoning in the Contemporary World, 2: Focus Questions for the Numeracy Community." Numeracy 3 (2): Article 5.

Dingman and Madison 2010

Steen and Madison 2011

Boersma et al. 2011

Boersma and Klyve 2013a

Boersma and Klyve 2013b (http://dx.doi.org/10.5038/1936-4660.3.2.4) (http://dx.doi.org/10.5038/1936-4660.4.1.1) (http://dx.doi.org/10.5038/1936-4660.4.2.8)

http://dx.doi.org/10.5038/1936-4660.6.1.5)

(http://dx.doi.org/10.5038/1936-4660.6.1.6) 
Polito 2014

Madison 2014

Russo 2015

Wismath and Worrall 2015 (http://dx.doi.org/10.5038/1936-4660.7.1.6) (http://dx.doi.org/10.5038/1936-4660.7.2.3) (http://dx.doi.org/10.5038/1936-4660.8.1.8) (http://dx.doi.org/10.5038/1936-4660.8.1.9)

Madison, Bernard L. and Lynn Arthur Steen. 2003. Quantitative Literacy: Why Numeracy Matters for Schools and Colleges. Princeton, NJ: The National Council on Education and the Disciplines. ${ }^{1}$

\begin{tabular}{|c|c|}
\hline Madison and Steen 2008 & (http://dx.doi.org/10.5038/1936-4660.1.1.2) \\
\hline Rheinlander et al. 2008 & (http://dx.doi.org/10.5038/1936-4660.1.1.4) \\
\hline Vacher and Chavez 2008 & (http://dx.doi.org/10.5038/1936-4660.1.2.2) \\
\hline Steele and Kiliç-Bahi 2008 & (http://dx.doi.org/10.5038/1936-4660.1.2.3) \\
\hline Madison and Steen 2009 & (http://dx.doi.org/10.5038/1936-4660.2.1.2) \\
\hline Wenner et al. 2009 & (http://dx.doi.org/10.5038/1936-4660.2.1.4) \\
\hline Grawe and Rutz 2009 & (http://dx.doi.org/10.5038/1936-4660.2.2.2) \\
\hline Mast 2009 & (http://dx.doi.org/10.5038/1936-4660.2.2.6) \\
\hline Grawe et al. 2010 & (http://dx.doi.org/10.5038/1936-4660.3.1.3) \\
\hline Sorey et al. 2010 & (http://dx.doi.org/10.5038/1936-4660.3.1.6) \\
\hline Dingman and Madison 2010 & (http://dx.doi.org/10.5038/1936-4660.3.2.4) \\
\hline Steen and Madison 2011 & (http://dx.doi.org/10.5038/1936-4660.4.1.1) \\
\hline Wetzel 2011 & (http://dx.doi.org/10.5038/1936-4660.4.1.4) \\
\hline Kosko and Wilkins 2011 & (http://dx.doi.org/10.5038/1936-4660.4.2.3) \\
\hline Sikorskii et al. 2011 & (http://dx.doi.org/10.5038/1936-4660.4.2.5) \\
\hline Boersma et al. 2011 & (http://dx.doi.org/10.5038/1936-4660.4.2.8) \\
\hline Sundre et al. 2012 & (http://dx.doi.org/10.5038/1936-4660.5.1.4) \\
\hline Hillyard 2012 & (http://dx.doi.org/10.5038/1936-4660.5.2.2) \\
\hline Smit and Mji 2012 & (http://dx.doi.org/10.5038/1936-4660.5.2.4) \\
\hline Mayes et al., 2013 & (http://dx.doi.org/10.5038/1936-4660.6.1.4) \\
\hline Boersma and Klyve 2013b & (http://dx.doi.org/10.5038/1936-4660.6.1.6) \\
\hline Rocconi et al. 2013 & (http://dx.doi.org/10.5038/1936-4660.6.2.10) \\
\hline Madison 2014 & (http://dx.doi.org/10.5038/1936-4660.7.2.3) \\
\hline Mayes et al., 2014 & (http://dx.doi.org/10.5038/1936-4660.7.2.5) \\
\hline Russo 2015 & (http://dx.doi.org/10.5038/1936-4660.8.1.8) \\
\hline Mayes et al. 2015 & (http://dx.doi.org/10.5038/1936-4660.8.2.4) \\
\hline Todd and Wagaman 2015 & (http://dx.doi.org/10.5038/1936-4660.8.2.9) \\
\hline Tunstall and Bossé 2015 & (http://dx.doi.org/10.5038/1936-4660.8.2.10) \\
\hline Tunstall 2015 & $\mathrm{v} \cdot / / \mathrm{dx}$ doi oro $/ 10-5038 / 1936-4660.821$ \\
\hline
\end{tabular}

Madison, Bernard L., and Lynn Arthur Steen. 2008. "Evolution of Numeracy and the National Numeracy Network." Numeracy 1 (1), Article 2.

$\begin{array}{ll}\text { Vacher and Chavez 2008 } & (\text { http://dx.doi.org/10.5038/1936-4660.1.2.2) } \\ \text { Boersma and Willard 2008 } & \text { (http://dx.doi.org/10.5038/1936-4660.1.2.5) } \\ \text { Karaali 2008 } & \text { (http://dx.doi.org/10.5038/1936-4660.1.2.6) } \\ \text { Sorey et al. 2010 } & \text { (http://dx.doi.org/10.5038/1936-4660.3.1.6) } \\ \text { Catalano 2010 } & \text { (http://dx.doi.org/10.5038/1936-4660.3.1.7) } \\ \text { Dingman and Madison 2010 } & \text { (http://dx.doi.org/10.5038/1936-4660.3.2.4) }\end{array}$

${ }^{1}$ Available at: http://www.maa.org/external_archive/QL/qltoc.html (accessed Nov 2, 2016) 
Vacher and Lardner 2010

Kosko and Wilkins 2011

Ward et al. 2011

Boersma et al. 2011

Madison 2012

Hillyard 2012

Boersma and Klyve 2013b

Harrison 2014

Madison 2014

Russo 2015

Dumford and Rocconi 2015

Madison 2015

Tunstall and Bossé 2015 (http://dx.doi.org/10.5038/1936-4660.3.2.6) (http://dx.doi.org/10.5038/1936-4660.4.2.3) (http://dx.doi.org/10.5038/1936-4660.4.2.4) (http://dx.doi.org/10.5038/1936-4660.4.2.8) (http://dx.doi.org/10.5038/1936-4660.5.1.6) (http://dx.doi.org/10.5038/1936-4660.5.2.2) (http://dx.doi.org/10.5038/1936-4660.6.1.6) (http://dx.doi.org/10.5038/1936-4660.7.2.2) (http://dx.doi.org/10.5038/1936-4660.7.2.3) (http://dx.doi.org/10.5038/1936-4660.8.1.8) (http://dx.doi.org/10.5038/1936-4660.8.1.5) (http://dx.doi.org/10.5038/1936-4660.8.1.11) (http://dx.doi.org/10.5038/1936-4660.8.2.10)

Madison, Bernard L., and Lynn Arthur Steen, eds. 2008. Calculation vs. Context: Quantitative Literacy and Its Implications for Teacher Education. Washington DC: Mathematical Association of America. ${ }^{2}$

Vacher and Chavez 2008

Madison and Steen 2009

Vacher and Chavez 2009

Lutsky 2009

Mast 2009

Grawe et al. 2010

Sorey et al. 2010

Dingman and Madison 2010

Vacher and Lardner 2010

Gaze 2010

Steen and Madison 2011

Watson 2011

Boersma et al. 2011

Madison 2012

Hillyard 2012

Maclellan 2012

Boersma and Klyve 2013b

Rocconi et al. 2013

Scharnagl et al. 2014

Polito 2014

Madison 2014

Mayes et al. 2014

Dumford and Rocconi 2015

Wright and Howard 2015

Russo 2015

Follette et al. 2015

Tunstall and Bossé 2015 (http://dx.doi.org/10.5038/1936-4660.1.2.2)

(http://dx.doi.org/10.5038/1936-4660.2.1.2)

(http://dx.doi.org/10.5038/1936-4660.2.1.5)

(http://dx.doi.org/10.5038/1936-4660.2.1.6)

(http://dx.doi.org/10.5038/1936-4660.2.2.6)

(http://dx.doi.org/10.5038/1936-4660.3.1.3)

(http://dx.doi.org/10.5038/1936-4660.3.1.6)

(http://dx.doi.org/10.5038/1936-4660.3.2.4)

(http://dx.doi.org/10.5038/1936-4660.3.2.6)

(http://dx.doi.org/10.5038/1936-4660.3.2.8)

(http://dx.doi.org/10.5038/1936-4660.4.1.1)

(http://dx.doi.org/10.5038/1936-4660.4.1.2)

(http://dx.doi.org/10.5038/1936-4660.4.2.8)

(http://dx.doi.org/10.5038/1936-4660.5.1.6)

(http://dx.doi.org/10.5038/1936-4660.5.2.2)

(http://dx.doi.org/10.5038/1936-4660.5.2.3)

(http://dx.doi.org/10.5038/1936-4660.6.1.6)

(http://dx.doi.org/10.5038/1936-4660.6.2.10)

(http://dx.doi.org/10.5038/1936-4660.7.1.4)

(http://dx.doi.org/10.5038/1936-4660.7.1.6)

(http://dx.doi.org/10.5038/1936-4660.7.2.3)

(http://dx.doi.org/10.5038/1936-4660.7.2.5)

(http://dx.doi.org/10.5038/1936-4660.8.1.5)

(http://dx.doi.org/10.5038/1936-4660.8.1.6)

(http://dx.doi.org/10.5038/1936-4660.8.1.8)

(http://dx.doi.org/10.5038/1936-4660.8.2.2)

(http://dx.doi.org/10.5038/1936-4660.8.2.10)

\footnotetext{
${ }^{2}$ Available at http://www.maa.org/sites/default/files/pdf/QL/cvc/CalcVsContext.pdf (accessed Nov 2, 2016)
} 
Madison, Bernard L., and Lynn Arthur Steen. 2009. "Confronting Challenges, Overcoming Obstacles: A Conversation about Quantitative Literacy.” Numeracy 2 (1): Article 2.

Grawe and Rutz 2009

(http://dx.doi.org/10.5038/1936-4660.2.2.2)

Frith 2012

Hillyard 2012

Russo 2015

(http://dx.doi.org/10.5038/1936-4660.5.1.3)

(http://dx.doi.org/10.5038/1936-4660.5.2.2)

(http://dx.doi.org/10.5038/1936-4660.8.1.8)

Madison, B., S. Boersma, C. Diefenderfer, and S. Dingman. 2009 [2012]. Case Studies for Quantitative Reasoning: A Casebook of Media Articles, $2^{\text {nd }}$ ed. [ $3^{\text {rd }}$ ed.] New York: Pearson Custom Publishing

Dingman and Madison $2010 \quad$ (http://dx.doi.org/10.5038/1936-4660.3.2.4)

Madison and Dingman $2010 \quad$ (http://dx.doi.org/10.5038/1936-4660.3.2.5)

Steen and Madison $2011 \quad$ (http://dx.doi.org/10.5038/1936-4660.4.1.1)

Boersma et al. $2011 \quad$ (http://dx.doi.org/10.5038/1936-4660.4.2.8)

Madison $2012 \quad$ (http://dx.doi.org/10.5038/1936-4660.5.1.6)

Boersma and Klyve 2013a $\quad$ http://dx.doi.org/10.5038/1936-4660.6.1.5)

Boersma and Klyve 2013b (http://dx.doi.org/10.5038/1936-4660.6.1.6)

Madison $2014 \quad$ (http://dx.doi.org/10.5038/1936-4660.7.2.3)

Wang and Wilder $2015 \quad$ (http://dx.doi.org/10.5038/1936-4660.8.2.6)

Wang 2015 (http://dx.doi.org/10.5038/1936-4660.8.2.7)

Mayfield and Dunham 2015 (http://dx.doi.org/10.5038/1936-4660.8.2.8)

Tunstall and Bossé $2015 \quad$ (http://dx.doi.org/10.5038/1936-4660.8.2.10)

Tunstall 2015

(http://dx.doi.org/10.5038/1936-4660.8.2.12)

Murray, Frank B. 2008. "The Licensure of Teachers for Quantitative Literacy: Who Should Be Entitled to Teach QL.” In Madison and Steen 2008: 163-185. Gaze $2010 \quad$ (http://dx.doi.org/10.5038/1936-4660.3.2.8)

Nelson, George D. 2003. "Quantitative Literacy: A Science Literacy Perspective." In Madison and Steen 2003: 179-180.

Sorey et al. $2010 \quad$ (http://dx.doi.org/10.5038/1936-4660.3.1.6)

Orrill, Robert. 2008. "Humanism and Quantitative Literacy." In Madison and Steen 2008: 47-57.

Madison 2012 (http://dx.doi.org/10.5038/1936-4660.5.1.6)

Richardson, Randall M. and William G. McCallum. 2003. "The Third R in Literacy." In Madison and Steen 2003: 99-106.

Steele and Kiliç-Bahi $2008 \quad$ (http://dx.doi.org/10.5038/1936-4660.1.2.3)

Wenner et al. $2009 \quad$ (http://dx.doi.org/10.5038/1936-4660.2.1.4)

Grawe and Rutz 2009 (http://dx.doi.org/10.5038/1936-4660.2.2.2)

Grawe et al. $2010 \quad$ (http://dx.doi.org/10.5038/1936-4660.3.1.3)

Wetzel $2011 \quad$ (http://dx.doi.org/10.5038/1936-4660.4.1.4)

Hillyard $2012 \quad$ (http://dx.doi.org/10.5038/1936-4660.5.2.2)

Russo 2015 (http://dx.doi.org/10.5038/1936-4660.8.1.8)

Tunstall and Bossé $2015 \quad$ (http://dx.doi.org/10.5038/1936-4660.8.2.10) 
Rosen, Linda, Lindsay Weil, and Claus von Zastrow. 2003. "Quantitative Literacy in the Workplace: Making It a Reality." In Madison and Steen 2003: 43-52. Mast 2009 (http://dx.doi.org/10.5038/1936-4660.2.2.6)

Scheaffer, Richard. L. 2003. "Statistics and Quantitative Literacy." In Madison and L.A. Steen 2003: 145-152. Madison and Steen $2008 \quad$ (http://dx.doi.org/10.5038/1936-4660.1.1.2)

Schield, Milo. 2008. "Quantitative Literacy and School Mathematics: Percentages and Fractions." In Madison and Steen 2008: 87-107.

Vacher and Chavez $2009 \quad$ (http://dx.doi.org/10.5038/1936-4660.2.1.5)

Grawe et al. $2010 \quad$ (http://dx.doi.org/10.5038/1936-4660.3.1.3)

Watson $2011 \quad$ (http://dx.doi.org/10.5038/1936-4660.4.1.2)

Madison $2012 \quad$ (http://dx.doi.org/10.5038/1936-4660.5.1.6)

Scharnagl et al. $2014 \quad$ (http://dx.doi.org/10.5038/1936-4660.7.1.4)

Polito $2014 \quad$ (http://dx.doi.org/10.5038/1936-4660.7.1.6)

Shavelson, Richard J. 2008. "Reflections on Quantitative Reasoning: An Assessment Perspective." In Madison and Steen 2008: 27-44.

Dingman and Madison $2010 \quad$ (http://dx.doi.org/10.5038/1936-4660.3.2.4)

Boersma et al. $2011 \quad$ (http://dx.doi.org/10.5038/1936-4660.4.2.8)

Madison $2012 \quad$ (http://dx.doi.org/10.5038/1936-4660.5.1.6)

Rocconi et al. $2013 \quad$ (http://dx.doi.org/10.5038/1936-4660.6.2.10)

Mayes et al., $2013 \quad$ (http://dx.doi.org/10.5038/1936-4660.6.1.4)

Mayes et al. $2014 \quad$ (http://dx.doi.org/10.5038/1936-4660.7.2.5)

Dumford and Rocconi $2015 \quad$ (http://dx.doi.org/10.5038/1936-4660.8.1.5)

Steen, Lynn Arthur. 2008. "Reflections on Wingspread Workshop." In Madison and Steen 2008: 11-23.

Vacher and Chavez $2009 \quad$ (http://dx.doi.org/10.5038/1936-4660.2.1.5)

Grawe et al. $2010 \quad$ (http://dx.doi.org/10.5038/1936-4660.3.1.3)

Gaze $2010 \quad$ (http://dx.doi.org/10.5038/1936-4660.3.2.8)

Watson $2011 \quad$ (http://dx.doi.org/10.5038/1936-4660.4.1.2)

Steen, Lynn Arthur and Bernard L. Madison. 2011. "Reflections on the Tenth Anniversary of Mathematics and Democracy." Numeracy, 4 (1): Article 1. Smit and Mji $2012 \quad$ (http://dx.doi.org/10.5038/1936-4660.5.2.4)

Taylor, Corrine. 2008. "Preparing Students for the Business of the Real (and Highly Quantitative) World.” In Madison and Steen 2008: 109-124.

Grawe et al. 2010

Sorey et al. 2010

Vacher and Lardner 2010

Gaze 2010

Madison 2012

Rocconi et al. 2013 (http://dx.doi.org/10.5038/1936-4660.3.1.3)

(http://dx.doi.org/10.5038/1936-4660.3.1.6)

(http://dx.doi.org/10.5038/1936-4660.3.2.6)

(http://dx.doi.org/10.5038/1936-4660.3.2.8)

(http://dx.doi.org/10.5038/1936-4660.5.1.6)

(http://dx.doi.org/10.5038/1936-4660.6.2.10) 
Tucker, Alan. 2008. "Fractions and Units in Everyday Life." In Madison and Steen 2008: 75-86.

Vacher and Chavez $2009 \quad$ (http://dx.doi.org/10.5038/1936-4660.2.1.5) Scharnagl et al. $2014 \quad$ (http://dx.doi.org/10.5038/1936-4660.7.1.4)

Wiggins, Grant. 2003. "Get Real!': Assessing for Quantitative Literacy.” In Madison and Steen 2003: 121-143.

Grawe et al. 2010

Boersma et al. 2011

Boersma and Klyve 2013b

Tunstall and Bossé 2015 (http://dx.doi.org/10.5038/1936-4660.3.1.3) (http://dx.doi.org/10.5038/1936-4660.4.2.8) (http://dx.doi.org/10.5038/1936-4660.6.1.6) (http://dx.doi.org/10.5038/1936-4660.8.2.10)

\section{Part 2: Source List (The Citing Papers)}

Boersma, Stuart, and Dominic Klyve. 2013a. "Using a Media-article Approach to Quantitative Reasoning as an Honors Course: An Exploratory Study." Numeracy 6 (1): Article 5. http://dx.doi.org/10.5038/1936-4660.6.1.5.

Boersma, Stuart, and Dominic Klyve. 2013b. "Measuring Habits of Mind: Toward a Prompt-Less Instrument for Assessing Quantitative Literacy." Numeracy 6 (1): Article 6. http://dx.doi.org/10.5038/1936-4660.6.1.6.

Boersma, Stuart, and Teri Willard. 2008. "False Positives and Referral Bias: Content for a Quantitative Literacy Course." Numeracy 1 (2): Article 5. http://dx.doi.org/10.5038/1936-4660.1.2.5.

Boersma, Stuart, Caren Diefenderfer, Shannon W. Dingman, and Bernard L. Madison. 2011. "Quantitative Reasoning in the Contemporary World, 3: Assessing Student Learning." Numeracy 4 (2): Article 8. http://dx.doi.org/10.5038/1936-4660.4.2.8.

Catalano, Michael T. 2010. "College Algebra in Context: A Project Incorporating Social Issues." Numeracy 3 (1): Article 7. http://dx.doi.org/10.5038/1936-4660.3.1.7.

Dingman, Shannon W., and Bernard L. Madison. 2010. "Quantitative Reasoning in the Contemporary World, 1: The Course and Its Challenges." Numeracy 3 (2): Article 4. http://dx.doi.org/10.5038/1936-4660.3.2.4.

Dumford, Amber D., and Louis M. Rocconi. 2015. "Development of the Quantitative Reasoning Items on the National Survey of Student Engagement." Numeracy 8 (1): Article 5. http://dx.doi.org/10.5038/1936-4660.8.1.5.

Follette, Katherine B., Donald W. McCarthy, Erin Dokter, Erin, Sanlyn Buxner, and Edward Prather. 2015. "The Quantitative Reasoning for College Science (QuaRCS) Assessment, 1: Development and Validation.” Numeracy 8 (2): Article 2. http://dx.doi.org/10.5038/1936-4660.8.2.2.

Frith, Vera. 2012. "Quantitative Literacy Interventions at University of Cape Town: Effects of Separation from Academic Disciplines." Numeracy 5 (1): Article 3. http://dx.doi.org/10.5038/1936-4660.5.1.3. 
Gaze, Eric. 2010. “Creating a Masters in Numeracy Program.” Numeracy 3 (2): Article 8. http://dx.doi.org/10.5038/1936-4660.3.2.8.

Grawe, Nathan D. 2013. "Does Completion of Quantitative Courses Predict Better Quantitative Reasoning-in-Writing Proficiency?” Numeracy 6 (2): Article 11. http://dx.doi.org/10.5038/1936-4660.6.2.11.

Grawe, Nathan D., and Carol A. Rutz. 2009. "Integration with Writing Programs: A Strategy for Quantitative Reasoning Program Development." Numeracy 2 (2): Article 2. http://dx.doi.org/10.5038/1936-4660.2.2.2.

Grawe, Nathan D., Neil S. Lutsky, and Christopher J. Tassava. 2010. "A Rubric for Assessing Quantitative Reasoning in Written Arguments." Numeracy 3 (1): Article 3. http://dx.doi.org/10.5038/1936-4660.3.1.3.

Harrison, Steven. 2014. "History of Numeracy Education and Training for Print Journalists in England." Numeracy 7 (2): Article 2. http://dx.doi.org/10.5038/19364660.7.2.2.

Hillyard, Cinnamon. 2012. "Comparative Study of the Numeracy Education and Writing Across the Curriculum Movements: Ideas for Future Growth." Numeracy 5 (2): Article 2. http://dx.doi.org/10.5038/1936-4660.5.2.2.

Howington, Heather. Thomas Hartfield, and Cinnamon Hillyard. 2015. "Faculty Viewpoints on Teaching Quantway®." Numeracy 8 (1): Article 10. http://dx.doi.org/10.5038/1936-4660.8.1.10.

Karaali, Gizem. 2008. "Word Problems: Reflections on Embedding Quantitative Literacy in a Calculus Course." Numeracy: 1 (2): Article 6. http://dx.doi.org/10.5038/1936-4660.1.2.6.

Kosko, Karl W., and Jesse L. Wilkins, Jesse L. 2011. "Communicating Quantitative Literacy: An Examination of Open-Ended Assessment Items in TIMSS, NALS, IALS, and PISA." Numeracy 4 (2): Article 3. http://dx.doi.org/10.5038/19364660.4.2.3.

Lutsky, Neil. 2009. "Review of The Chicago Guide to Writing about Numbers by Jane E. Miller." Numeracy 2 (1): Article 6. http://dx.doi.org/10.5038/1936-4660.2.1.6.

Maclellan, Effie. 2012. "Number Sense: The Underpinning Understanding for Early Quantitative Literacy.” Numeracy 5 (2): Article 3. http://dx.doi.org/10.5038/1936-4660.5.2.3.

Madison, Bernard L. 2009. "'All the More Reason for QR Across the Curriculum." Numeracy 2 (1): Article 1. http://dx.doi.org/10.5038/1936-4660.2.1.1.

Madison, Bernard L. 2012. "If Only Math Majors Could Write..." Numeracy 5 (1): Article 6. http://dx.doi.org/10.5038/1936-4660.5.1.6.

Madison, Bernard L. 2014. "How Does One Design or Evaluate a Course in Quantitative Reasoning?” Numeracy 7 (2): Article 3. http://dx.doi.org/10.5038/1936-4660.7.2.3.

Madison, Bernard L. 2015. "Quantitative Literacy and the Common Core State Standards in Mathematics." Numeracy 8 (1): Article 11.

http://dx.doi.org/10.5038/1936-4660.8.1.11. 
Madison, Bernard L., and Shannon W. Dingman. 2010. "Quantitative Reasoning in the Contemporary World, 2: Focus Questions for the Numeracy Community." Numeracy 3 (2): Article 5. http://dx.doi.org/10.5038/1936-4660.3.2.5.

Madison, Bernard L., and Lynn Arthur Steen. 2008. "Evolution of Numeracy and the National Numeracy Network." Numeracy 1 (1): Article 2. http://dx.doi.org/10.5038/1936-4660.1.1.2.

Madison, Bernard L., and Lynn Arthur Steen. 2009. "Confronting Challenges, Overcoming Obstacles: A Conversation about Quantitative Literacy." Numeracy 2 (1): Article 2. http://dx.doi.org/10.5038/1936-4660.2.1.2.

Mast, Maura B. 2009. "Review of Calculation vs. Context: Quantitative Literacy and Its Implications for Teacher Education by Bernard L. Madison and Lynn Arthur Steen (Editors)." Numeracy 2 (2): Article 6. http://dx.doi.org/10.5038/1936-4660.2.2.6.

Mayes, Robert L., Franziska Peterson, and Rachel Bonilla. 2013. "Quantitative reasoning learning progressions for environmental science: Developing a framework." Numeracy 6 (1): Article 4. http://dx.doi.org/10.5038/1936-4660.6.1.4.

Mayes, Robert L., Jennifer Forrester, Jennifer Schuttlefield Christus, Franziska Peterson, and Rachel Walker. 2014. "Quantitative Reasoning Learning Progression: The Matrix.” Numeracy 7 (2): Article 5. http://dx.doi.org/10.5038/1936-4660.7.2.5.

Mayes, Robert L., Kent Rittschof, Jennifer H. Forrester, Jennifer D. Schuttlefield Christus, Lisa Watson, Lisa, and Franziska Peterson. 2015. "Quantitative Reasoning in Environmental Science: Rasch Measurement to Support QR Assessment." Numeracy 8 (2): Article 4. http://dx.doi.org/10.5038/1936-4660.8.2.4.

Mayfield, Betty, and Jill B. Dunham. 2015. "Adapting to a New Core Curriculum at Hood College: From Computation to Quantitative Literacy." Numeracy 8 (2): Article 8. http://dx.doi.org/10.5038/1936-4660.8.2.8.

Polito, Jessica. 2014. "The Language of Comparisons: Communicating about Percentages." Numeracy 7 (1): Article 6. http://dx.doi.org/10.5038/1936-4660.7.1.6. Rheinlander, Kim, Dorothy Wallace, Wells Morrison, Daniel Ansari, Donna Coch, and B. Venus Williams. 2008. "Teachers Talk: Pressure Points in the K-8 Mathematics Curriculum.” Numeracy 1 (1): Article 4. http://dx.doi.org/10.5038/1936-4660.1.1.4.

Ricchezza, Victor J., and H. L. Vacher. 2015. "Review of Developing Quantitative Literacy Skills in History and the Social Sciences: A Web-Based Common Core Approach by Kathleen W. Craver." Numeracy 8 (2): Article 14. http://dx.doi.org/10.5038/1936-4660.8.2.14.

Rocconi, Louis M., Amber D. Lambert, Alexander C. McCormick, and Shimon A. Sarraf. 2013. "Making College Count: An Examination of Quantitative Reasoning Activities in Higher Education.” Numeracy 6 (2): Article 10. http://dx.doi.org/10.5038/1936-4660.6.2.10.

Russo, Mark. 2015. "Quantitative Literacy and Co-Construction in a High School Math Course.” Numeracy 8 (1): Article 8. http://dx.doi.org/10.5038/1936-4660.8.1.8. 
Scharnagl, Susanne, Petra Evanschitzky, Judith Streb, Manfred Spitzer, and Katrin Hille. 2014. "Sixth Graders Benefit from Educational Software When Learning About Fractions: A Controlled Classroom Study." Numeracy 7(1): Article 4. http://dx.doi.org/10.5038/1936-4660.7.1.4.

Sikorskii, Alla, Vince Melfi, Dennis Gilliland, Jennifer Kaplan, and Suzie Ahn. 2011. "Quantitative Literacy at Michigan State University, 1: Development and Initial Evaluation of the Assessment." Numeracy 4 (2): Article 5. http://dx.doi.org/10.5038/1936-4660.4.2.5.

Smit, Antonie Christoffel, and Andile Mji. 2012. "Assessment of Numeracy Levels of Mine Workers in South African Chrome Mines.” Numeracy 5(2): Article 4. http://dx.doi.org/10.5038/1936-4660.5.2.4.

Sorey, Timothy L., Teri Willard, and Duane Sholz. 2010. “An Activity Promoting the Practice of Quantitative Literacy for Pre- and In-Service Teachers of Mathematics and Science." Numeracy 3(1): Article 6. http://dx.doi.org/10.5038/1936-4660.3.1.6.

Steele, Benjamin, and Semra Kiliç-Bahi. 2008. "Quantitative Literacy Across the Curriculum: A case study." Numeracy 1 (2): Article 3. http://dx.doi.org/10.5038/1936-4660.1.2.3.

Steele, Benjamin, and Semra Kiliç-Bahi. 2010. "Quantitative Literacy: Does It Work? Evaluation of Student Outcomes at Colby-Sawyer College." Numeracy 3 (2): Article 3. http://dx.doi.org/10.5038/1936-4660.3.2.3.

Steen, Lynn Arthur, and Bernard L. Madison. 2011. "Reflections on the Tenth Anniversary of Mathematics and Democracy." Numeracy 4 (1): Article 1. http://dx.doi.org/10.5038/1936-4660.4.1.1.

Sundre, Donna, Carol Barry, Vidar Gynnild, and Erin Tangen Ostgard. 2012. "Motivation for Achievement and Attitudes Toward Mathematics Instruction in a Required Calculus Course at the Norwegian University of Science and Technology." Numeracy 5 (1): Article 4. http://dx.doi.org/10.5038/1936-4660.5.1.4.

Todd, Vicki, and John Wagaman. 2015. "Implementing Quantitative Literacy at Southwestern Community College, North Carolina." Numeracy 8 (2): Article 9. http://dx.doi.org/10.5038/1936-4660.8.2.9.

Tunstall, Samuel L. 2015. "Review of Case Studies for Quantitative Reasoning: A Casebook of Media Articles by Bernard L. Madison, Stuart Boersma, Caren L. Diefenderfer, and Shannon W. Dingman." Numeracy 8 (2): Article 12. http://dx.doi.org/10.5038/1936-4660.8.2.12.

Tunstall, Samuel L., and Michael J. Bossé. 2015. "Promoting Numeracy in an Online College Algebra Course Through Projects and Discussions." Numeracy 8 (2): Article 10. http://dx.doi.org/10.5038/1936-4660.8.2.10.

Vacher, H. L. 2014. "Looking at the Multiple Meanings of Numeracy, Quantitative Literacy, and Quantitative Reasoning." Numeracy 7 (2): Article 1. http://dx.doi.org/10.5038/1936-4660.7.2.1. 
Vacher, H. L., and Todd Chavez. 2008. "Quantitative Literacy on the Web of Science, 1: The Bibliography and Its Role in the History of This Journal." Numeracy 1 (2):

Article 2. http://dx.doi.org/10.5038/1936-4660.1.2.2.

Vacher, H. L., and Todd Chavez. 2009. "Quantitative Literacy on the Web of Science, 2 - Mining the Health Numeracy Literature for Assessment Items." Numeracy 2 (1): Article 5. http://dx.doi.org/10.5038/1936-4660.2.1.5.

Vacher, H. L., and Emily Lardner. 2010. "Spreadsheets Across the Curriculum, 1: The Idea and the Resource." Numeracy 3 (2): Article 6. http://dx.doi.org/10.5038/19364660.3.2.6.

Wang, Frank. 2015. "Numeracy Infusion Course for Higher Education (NICHE), 2: Development of Students' Bayesian Reasoning Skill." Numeracy 8 (2): Article 7. http://dx.doi.org/10.5038/1936-4660.8.2.7.

Wang, Frank, and Esther I. Wilder. 2015. "Numeracy Infusion Course for Higher Education (NICHE), 1: Teaching Faculty How to Improve Students' Quantitative Reasoning Skills Through Cognitive Illusions." Numeracy 8 (2): Article 6. http://dx.doi.org/10.5038/1936-4660.8.2.6.

Ward, Rose Marie, Monica C. Schneider, and James D. Kiper. 2011. "Development of an Assessment of Quantitative Literacy for Miami University." Numeracy 4 (2): Article 4. http://dx.doi.org/10.5038/1936-4660.4.2.4.

Watson, Jane M. 2011. "Personal and Professional Numeracy: A Unit for Pre-Service Teachers at the University of Tasmania." Numeracy 4 (1): Article 2. http://dx.doi.org/10.5038/1936-4660.4.1.2.

Wenner, Jennifer M., Eric M. Baer, Cathryn A. Manduca, R. Heather Macdonald, Samuel Patterson, and Mary Savina. 2009. "The Case for Infusing Quantitative Literacy into Introductory Geoscience Courses." Numeracy 2 (1): Article 4. http://dx.doi.org/10.5038/1936-4660.2.1.4.

Wetzel, Laura Reiser. 2011. "Spreadsheets Across the Curriculum, 2: Assessing Our Success with Students at Eckerd College." Numeracy 4 (1): Article 4. http://dx.doi.org/10.5038/1936-4660.4.1.4.

Whitaker, Douglas, Steven Foti, and Tim Jacobbe. 2015. "The Levels of Conceptual Understanding in Statistics (LOCUS) Project: Results of the Pilot Study." Numeracy 8 (2): Article 3. http://dx.doi.org/10.5038/1936-4660.8.2.3.

Wismath, Shelly L., and Alyson Worrall. 2015. "Improving University Students' Perception of Mathematics and Mathematics Ability." Numeracy 8 (1): Article 9. http://dx.doi.org/10.5038/1936-4660.8.1.9.

Wright, Mary C. and Joseph E. Howard. 2015. "Assessment for Improvement: Two Models for Assessing a Large Quantitative Reasoning Requirement." Numeracy 8 (1): Article 6. http://dx.doi.org/10.5038/1936-4660.8.1.6. 